\section{AB0018 B CELL DISTURBANCE IN RHEUMATOID ARTHRITIS PATIENTS: COMPARATIVE STUDY BETWEEN TREATED AND NON-TREATED PATIENTS}

M.H. Nassr ${ }^{1}$, E. Gomaa ${ }^{2}$, M.M. Eid ${ }^{3}$, A.A. Hamdy ${ }^{2}$, A.A. Wegdan ${ }^{2}$ ${ }^{1}$ Rheumatology department; ${ }^{2}$ Microbiology and Immunology; ${ }^{3}$ Clinical Pathology Department, Fayoum University, Fayoum, Egypt

Background: B cells have been shown to play a key role in the pathogenesis of rheumatoid arthritis (RA). Alterations of B cell homeostasis as well as B cell activation markers, such as B-cell activating factor (BAFF) and A ProliferationInducing Ligand (APRIL) have been described in RA patients. Up to our knowledge, no data is currently available on differences between treatment naïve patients and those receiving disease modifying anti-rheumatic drugs (DMARDs), regarding B-cell surface expression as well as levels of circulating BAFF/APRIL. Objectives: The aim of this study is to investigate disturbance of $B$ cell as well as B cell activation markers namely BAFF and APRIL in patients with rheumatoid arthritis comparing treatment naive patients with those receiving disease modifying anti-rheumatic drugs.

Methods: Sixty RA patients and 30 healthy controls were enrolled. Thirty patients receiving non biologic DMARDs and had not received prior biological treatment and 30 treatment naïve patients. Absolute number of blood CD19 B cells was determined by flow cytometry using the CD19-PE Kit (Immunotech, France). BAFF and APRIL blood concentration was measured using commercially available ELISA kits (Bosterbio, USA).

Results: There was statistically significant difference $(p$-value $<0.05)$ between different study groups as regard B cell count with low mean among treated

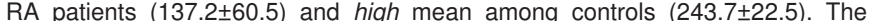
$B$ cell count was diminished in the two groups of RA patients, however it is more pronounced in treated patients. Circulating BAFF levels were increased in RA compared to $\mathrm{HC}$ ( $\mathrm{p}$-value <0.05) with more increase in patients on treatment. Circulating APRIL levels were significantly lower ( $p$-value $<0.05$ ) in treatment naïve rheumatoid arthritis patients $(343.9 \pm 21.7)$ than the control group (371.5 \pm 24.3$)$. However, there was no statistical significant difference ( $p$-value $>0.05$ ) between each of treated rheumatoid arthritis group and controls or with non treated rheumatoid arthritis group.

\begin{tabular}{llcc}
\hline Variables & \multicolumn{2}{c}{ B cell count } & p-value \\
\cline { 2 - 3 } & Mean & SD & \\
\hline G1: Treated RA patients & 137.2 & 60.5 & $<0.001$ \\
G2: Non treated patients & 179.3 & 59.1 & \\
G3: Controls & 243.7 & 22.5 & \\
\hline Variables & \multicolumn{2}{c}{ baff Concentration $(\mathrm{pg} / \mathrm{ml})$} & p-value \\
\cline { 2 - 3 } & Mean & SD & \\
\hline G1: Treated RA patients & 1419.4 & 417.3 & $<\mathbf{0 . 0 0 1}$ \\
G2: Non treated patients & 886.2 & 258.4 & \\
G3: Controls & 444.4 & 120.1 & \\
\hline
\end{tabular}

Conclusions: More decrease in B cell count and more increase in BAFF level were observed in RA patients receiving non-biologic DMARDs. Conversely, APRIL levels were not affected by treatment.

The contribution of B lymphocyte to RA pathogenesis goes beyond autoantibody production. Disturbances in B cell homeostasis in RA are not only due to disease process itself but closely related to the use of anti-rheumatic drugs.

\section{References:}

[1] Kotzin BL (2005) The role of B cells in the pathogenesis of rheumatoid arthritis. J Rheumatol Suppl 73: 14-18.

[2] Edwards JC, Cambridge G.(2006) B-cell targeting in rheumatoid arthritis and other autoimmune diseases. Nat Rev Immunol. May;6(5):394-403.

[3] Ng LG, Mackay CR, Mackay F (2005) The BAFF/APRIL system: life beyond B lymphocytes. Mol Immunol 42: 763-772.

[4] Vincent F., Saulep-Easton D., Figgett W., Fairfax K., Mackay F. (2013) The BAFF/APRIL system: emerging functions beyond B cell biology and autoimmunity. Cytokine Growth Factor Rev 24: 203-215.

Disclosure of Interest: None declared

DOI: 10.1136/annrheumdis-2017-eular.2014

\section{AB0019 HDL PROMOTES A REGULATORY PHENOTYPE OF THE IMMUNE SYSTEM BY INDUCING THE PROLIFERATION OF HUMAN CD4+ T CELLS WHILE INCREASING THE EXPRESSION OF FOXP3 IN REGULATOR T CELLS}

M. Fernandes Das Neves ${ }^{1,2}$, J.R. Batuca ${ }^{2}$, E.C. Jury ${ }^{3}$, J. Delgado Alves ${ }^{1,2}$ ${ }^{1}$ Medicine IV, Professor Doutor Fernando Fonseca Hospital, Amadora; ${ }^{2}$ CEDOC - Chronic Disease Research Center, NOVA Medical School/Faculdade de Ciências Médicas, Universidade Nova de Lisboa, Lisbon, Portugal: ${ }^{3}$ Centre for Rheumatology Research, University College London, London, United Kingdom

Background: High-density lipoproteins (HDL) are the plasma lipoproteins responsible for reverse cholesterol transport. Its protective effect on cardiovascular disease is attributed to the cholesterol efflux capacity as well as to its anti-oxidant and anti-inflammatory properties. HDL low levels are associated with immune dis- eases, including systemic lupus erythematosus and rheumatoid arthritis, implying a potential link between $\mathrm{HDL}$ and immunity.

There is few evidence on the effects of HDL on human T cells. Animal studies suggested that $\mathrm{HDL}$ inhibits lymphocyte proliferation ${ }^{1}$, but the only study in humans showed that HDL from young and healthy subjects increases the proliferation of lymphocytes ${ }^{2}$. Previous studies also showed that HDL levels are positively correlated with the prevalence of regulator T cells in peripheral blood ${ }^{3}$. Furthermore, it has been shown that one of the main actions of HDL in lymphocytes is the disaggregation of lipid rafts provoked by cholesterol efflux from the membrane, but the final consequences of this effect are not known.

Objectives: To determine the in vitro effects of HDL in human T cell proliferation and in the frequency of FoxP3+ regulator T cells.

Methods: Peripheral blood mononuclear cells from six healthy donors were cultured with and without HDL to measure membrane cholesterol and proliferative response to $\mathrm{CD} 3 / \mathrm{CD} 28$ stimulation. Membrane cholesterol was measured by filipin binding, assessed by flow cytometry. $\mathrm{T}$ cell proliferation was studied by $\mathrm{Ki}$ 67 expression and cell trace staining for multiple generations. T cell phenotyping and FoxP3 fluorescence were analyzed by flow cytometry.

Results: HDL significantly reduced the amount of membrane cholesterol in CD4+ $T$ cells, after 24 hours in culture. When added in the absence of stimulation, $\mathrm{HDL}$ did not induce any proliferation in CD4+ T cells. When added in conjunction with CD3/CD28 stimulation, HDL significantly increased the proliferation of CD4+ $T$ cells. $T$ cell phenotyping showed a significant increase in CD4+CD25+ FoxP3 fluorescence, after culture with HDL.

Conclusions: This study shows that HDL promotes cholesterol efflux from the membrane of CD4+ $\mathrm{T}$ cells in vitro and increases CD4+ $\mathrm{T}$ cell proliferation. However, the increase in $\mathrm{T}$ cell numbers seem to favor the expression of FoxP3 in regulator in $\mathrm{T}$ cells, which is associated with suppression of inflammation. With the development of therapies to increase HDL levels, the knowledge of the HDL effects on $T$ cell subsets is very important for the future management of cardiovascular and rheumatic diseases.

References:

[1] Wilhem AJ, Zabalawi M, Owen JS, et al. Apolipoprotein A-I modulates regulatory T cells in autoimmune LDLr-/-, ApoA-I-/- mice. J Biol Chem. 2010; 285:36158-36169.

[2] Larbi A, Fortin C, Dupuis G, et al. Immunomodulatory role of high-density lipoproteins: impact on immunosenescence. Age. 2014; 36(5):9712.

[3] Ammirati E, Cianflone D, Banfi M, et al. Circulating CD4+CD25hiCD127lo regulatory T-cell levels do not reflect the extent or severity of carotid and coronary atherosclerosis. Arterioscler Thromb Vasc Biol. 2010; 30:1832-1841.

Disclosure of Interest: None declared

DOI: 10.1136/annrheumdis-2017-eular.6681

\section{AB0020 EXPRESSION LEVELS OF SIGNALING LYMPHOCYTE ACTIVATION MOLECULE FAMILY 6 (SLAMF6) ON CIRCULATING FOLLICULAR HELPER T CELLS ARE ASSOCIATED WITH DISEASE ACTIVITY IN SYSTEMIC LUPUS ERYTHEMATOSUS}

S.E. Kang ${ }^{1}$, S.J. Lee ${ }^{1,2}$, D.J. Go ${ }^{1,2}$, H.M. Kwon ${ }^{2}$, E.Y. Ahn ${ }^{2}$, J.K. Park ${ }^{1,2}$ E.Y. Lee ${ }^{2}$, E.B. Lee ${ }^{2}$, Y.W. Song ${ }^{1,2} .{ }^{1}$ Department of Molecular Medicine and Biopharmaceutical Sciences, Graduate School of Convergence Science and Technology, Seoul National University; ${ }^{2}$ Division of Rheumatology, Department of Internal Medicine, Seoul National University Hospital, Seoul, Korea, Republic Of

Background: T follicular helper cells (Tfh) are necessary for B-cell maturation and differentiation in the germinal centers (GC). The signaling lymphocyte activation molecule family (SLAMF) receptors on the cell surface mediate $T$ cell:B cell interaction for formation and maintenance of GC. But it remains to be elucidated whether SLAMF is expressed in circulating T follicular helper cells (cTfh).

Objectives: The aim of this study was to investigate the levels of SLAMF3 and SLAMF6 on cTfh and to analyze their association with disease activity in systemic lupus erythematosus (SLE) patients.

Methods: Blood samples were collected from 50 SLE patients, 24 Sjogren's syndrome (SS) patients and 25 age- and sex-matched healthy controls (HCs). In this study, CXCR5, programmed cell death protein 1 (PD-1), and CD4 were used as markers to define cTfh in peripheral blood mononuclear cells (PBMCs) The expression levels of SLMAF3 and SLMAF6 on cTfh were compared to that of circulating $B$ cell subsets by flow cytometry. Clinical features including disease activity and laboratory tests were analyzed according to expression of SLAMF3 and SLAMF6.

Results: Surface expression of SLAMF6 on CD4 T cells was significantly increased in SLE patients compared to SS patients (mean fluorescence intensity [MFI], mean \pm SD: $1291 \pm 319$ vs $1098 \pm 147, \mathrm{p}=0.015)$ and HCs $(1291 \pm 319$ vs $1094 \pm 243, p=0.011)$. But there was no difference in expression of SLAMF3 on CD4 T cells between SLE patients and SS patients or HCs. SLAMF6 expressions on cTfh cells, identified as $\mathrm{CD} 4^{+} \mathrm{CXCR} 5^{+} \mathrm{PD}-1^{+}$, were significantly increased in SLE patients than in SS patients $(1500 \pm 270$ vs $1296 \pm 140, p=0.001)$ and HCs $(1500 \pm 270$ vs $1295 \pm 161, p=0.001)$. SLAMF6 expressions on cTfh cells had correlation with systemic lupus erythematosus disease activity index (SLEDAI) (Spearman's rho $=0.477, \mathrm{p}=0.001$ ), proteinuria ( $r \mathrm{ho}=0.321, \mathrm{p}=0.030$ ) and double strand DNA ( $r h o=0.340, p=0.026$ ) in SLE patients. Moreover, expression levels of SLAMF6 on cTfh cells were correlated with those of several B cell subsets including total $B$ cells $\left(C D 19^{+}\right)$, naïve $B$ cells $\left(C D 19^{+} C^{-} 27^{-} \lg D^{+}\right)$and class 